Nepal Journal of Science and Technology Vol. 14, No.2 (2013) 155-160

\title{
Morphology and AFM Spectroscopy of Irradiated Interface of Silicon
}

\author{
S. K. Lamichhane \\ Prithvi Narayan Campus, Trivhuvan University,Pokhara \\ e-mail: sklamichhane@hotmail.com
}

\begin{abstract}
In covalent solids, more energetic irradiation sources are necessary to produce detectable level of damage. The atomic force microscopic (AFM) studies of mega electron-volt (MeV) ions irradiated silicon surfaces have been studied to a fluence of $5 \times 10^{8}$ ions $\mathrm{cm}^{-2}$ and surface morphology has been studied with AFM. Interesting features of cracks of $\sim 50 \mathrm{~nm}$ in depth and $\sim 100 \mathrm{~nm}$ in width have been observed on the irradiated surface. The features seemed to have been caused by the irradiation-induced stress in the irradiated regions of the target surface. The observed feature of cracks seems to be mainly due to the high electronic energy loss of the irradiated ions on the surface induces the stress in it. It confirms that the coarseness of the microstructure of a material directly affects the mechanical properties.
\end{abstract}

Key words: irradiated silicon, AFM, pull-off force, piezoelectric, SOI

\section{Introduction}

Measurement of material property at an interface with structure in nanometer range is now emerging as an interesting topic both for basic and applied research. The theories of energy transfer by lattice displacement in solids are well documented in literature. In the literature Sen and Akhtar (2002) and Sen et al. (2000) have reported the interaction of high energy, mega electron volt $(\mathrm{MeV})$ ions with a single crystalline target material such as silicon, in such a manner that following a Coulomb explosion mechanism and shock wave formation, there is localization of vibrational energy. The imparted energy is transferred through the single crystalline lattice such that the chains of atoms form facile pathways aiding such energy transfer. The energy is deposited in predetermined regions of the crystalline lattice to form irradiation interfaces. Employing x-ray topography, these authors have established the formation of strain at these interfaces. These strained regions are of immense interest for the formation of artificial strained structures.

Investigation of these findings as the presence of irradiated interface on silicon surface is of main concern. As the stress applied by $\mathrm{MeV}$ is directly proportional to strain produced and hence the mechanical deformation would take place at irradiated interface region. Such finding motivates our attention whether one can develop a quantitative technique by which one can understand the presence of anisotropy and quantitative magnitude of strain that appears at irradiation interface.

Mechanical properties of materials can be studied through analysis of force-distance (F-D) curve generated with an atomic force microscope (AFM). AFM force-distance curves are obtained by monitoring the cantilever deflection as a function of the vertical displacement of the piezoelectric scanner. Laser based optical detection system has been used for cantilever deflection measurement in our system. By force-spectroscopy one can systematically determine the dependence of the interaction on probesample distance. The variation of the F-D spectrum in AFM is such that the far right side of the curve is defined to be where the scanner is fully retracted at which the force experienced by probe is null. As the probe approaches the sample surface, the force is increased in contact region. Following the way upon this point, the shape of the approach curve at this region may provide information on visco-elasticity of 
the sample (Sen et al. 1998). In addition to this, far below the null position there is a point in F-D curve where probe retracts from the sample surface is snapoff point. We address these two cases as minimum loaded and heavily loaded conditions of the cantilever respectively. The difference between the minimum and maximum loading condition of the cantilever is pulloff or adhesion force (Koleske et al. 1995). In other words, at some critical distance the tip feels an attractive force from the surface and falls quickly towards the surface until contact (Mizes et al. 1991) and sample motion bends the tip backwards. When the sample is retracted, the tip continues to touch the sample, even in post equilibrium position. The tip will adhere until the force extracted by the cantilever is enough to break the adhesion. The cantilever then springs back to the resting position. The difference in the minimum of retracting curve and the tip resting position is known as adhesion force. This situation reserves the potentiality to assess the surface energy of the region of the sample surface under consideration.

In this work, variation of pull-off force as measured by AFM has been presented in 2D plot summarizing the features that are present at the interface. Present work aims to provide better understanding of unusual variation of pull-off or adhesion and hence the nature of binding interaction that exists in irradiated interface. The pull-off force distribution in 2D plot has been analyzed across the length and width of the irradiated interface. Moreover, surface condition of the irradiated interface has been analyzed with the help of AFM images as well as corresponding plot of pull-off force. In brief, an effort has been made to use force spectroscopy as a tool for detection and characterization of material inhomogenities present at interface.

\section{Methodology}

Our region of interest was on the silicon surface where lack of irradiation through $\mathrm{MeV}$ ion energy loss would produce an irradiation interface, simply by creating a hot region (or region under irradiation) on one side and a cold region next to it. Location of the lattice site at cold region was spatially separated from the region of ion impact such that even induced $\mathrm{MeV}$ could not affect the protected cold region on sample surface. A spatial filtering was made by square grid composed of $\mathrm{Ni}$ wire having square cross section with $40 \mu \mathrm{m}$ edge length, at a spacing of $850 \mu \mathrm{m}$ was placed between 5 $\mathrm{mm}$ to $20 \mathrm{~cm}$ in front of the sample silicon. Choosing a wire here is quite important. Ni wire was chosen to be of thickness of approximately double the range of the $\mathrm{MeV}$ employed. This prevents any possibility of forward sputtering from the nickel grid into the silicon (100) crystal. Time of flight (TOF) and secondary mass spectroscopic (SMS) analysis was carried out on the sample before and after irradiation. After irradiation the surface of the crystal was treated with dash etchant to a depth of about $50 \mathrm{~nm}$ to remove sample contamination or damage.

The irradiation procedure reported here was carried out by using a 15 UD pelletron accelerator under a vacuum of $10^{-6}$ torr, at room temperature. The flux was kept at $5 \times 10^{8}$ ions $\mathrm{cm}^{-2} \mathrm{sec}$ and maximum affluence not exceeding $10^{11}$ ions $\mathrm{cm}^{-2} \mathrm{sec}^{2}$ during the experiment.

The sample was mounted on a piezoelectric tube, driven towards the tip under computer control for taking images. The motion of the tip was monitored as a function of sample displacement. Atomic force microscope (AFM) images in contact mode were recorded with Thermo Microscopes Autoprobe Electronics Module Model No. APEM 1000, CA USA. Before recording the data on spectroscopy mode, cantilever probe was fully calibrated as per its force constant. Using spectroscopy mode of Proscan Data Acquisition software, 20 different locations were chosen at the interface of the AFM images on irradiation interface. And finally 16 data points belonging to F-D response were recorded for each location.

\section{Results and Discussion}

Microstructural investigation are useful as they are capable of direct resolution of the defect structure. AFM reveal about the structure of the defected solids. Except in ultra high vacuum, the surfaces of the AFM tip and the samples were always covered, either partially or completely, with an adsorbed layer of molecules from the environment. Therefore, the interaction between the tip and the sample surface were mediated through these adsorbed species. On the other hand, often the tip and the sample surface are intentionally exposed to the chemical like $\mathrm{KOH}$, as demand by the design of the experiment. In these cases, the tip (or the sample) is said to be functionalized. 
It is generally believed that the displacement spike of $\mathrm{MeV}$ irradiation may involve a region of the crystal containing as many as $10^{6}$ atoms sites, at the centre of which are lattice vacancies with the interstitials being closer to the periphery of the spike (Lugomer et al. 2012). Owing to the thermal motion of the atoms, many interstitial vacancy pairs are spontaneously recombine, thereby reducing the amount of damage in the region of the displacement spike.

Microscopic studies have shown the increase of defects such as dislocation of loops and their tangling appearance. These dislocations are displaced in specific regions, due to the process of vacancies and interstitials, which increase their cumulation. Thus, different kinds of defects (dislocations and interstitials) and their complexes appeared during irradiation. The strongly damaged regions were imaged in the microscope as black (B) islands and the less damaged regions were imaged as white (W) islands. For all fluencies, the regions W with micro-hardness smaller than in non-irradiated silicon were observed by AFM as depicted in Figs. 1 and 2. Micro-hardness was larger in the regions where the concentration of dislocation loops was high. The $\mathrm{W}$, regions have a small number of the dislocation loops, and single punctual defects were seen through AFM. The dislocation loops were placed in specific B regions, which increase in size with the increase of fluence due to a process of vacancies and interstitials accumulation. Using an AFM it was found that the W regions contain smaller number of dislocation loops and a large amount of single point defects with their cumulations.

In Fig. 2, it is clearly seen that the surface has cracked in three directions, while in Fig. 3 the same feature has been clearly viewed (for the smaller scan size). The depth and height of the feature have been measured using the software available with the AFM and they were found to be of $\sim 47 \mathrm{~nm}$ and $\sim 103 \mathrm{~nm}$ respectively. The observed feature is due to the irradiation-induced stress in the target material. The electronic flux of $\mathrm{MeV}$ Si ions in silicon was found to be of $2.43 \mathrm{MeV} /$ micron, while the nuclear flux was $1.94 \mathrm{KeV} /$ micron. Since the electronic energy loss was maximum at the surface and the observed feature has the depth of 50 $\mathrm{nm}$ only, the observed features seems mainly due to electronic energy loss of the incident ions. The energy loss in the near-surface region seems to be high enough to lead the various effects in the irradiated region. It has been shown that (Erlandsson et al. 1988) once, amorphization takes place the in-plane stress as well as strain begins to decrease due to loss of energy thermally by the plane. The decrease of the in-plane strain may result in the flow of silicon atoms out of the target plane in the irradiated region. When amorphous materials are subjected to swift heavy ion beam irradiation, they behave like a visco-elastic fluid. Moreover, in_swift heavy ion irradiation, there is an occurrence of thermal spike (Martin \& Wickramasinghe 1987). This thermal spike leads to high local heating, which also leads to the observable effect. It has also been shown that the final state of material within a thermal spike is more disordered and thus strained with respect to the initial state. This strain gives rise to a stress even if the sample was originally stress-free, because of the constraints imposed by the un-irradiated surrounding matrix. As a result, an inclusion produced in the bulk is compressed to a size between its original volume and a stress-free transformation volume. The shape of the inclusion determines the anisotropy in the components of the constrained strain and that of elastic strain thus produced. In the case of a spherical inclusion, the stressfree and the constrained strain are hydrostatic in nature. Thus the deformation caused by the resulting elastic strain and stress, which are hydrostatic as well, are identical in all directions. However, for the ellipsoidal or cylindrical shape of the inclusion, the constrained strain and the elastic strain and stress produced in the inclusions have shear components too. If the length of an inclusion (l) is much larger than its diameter (d), i.e., $\mathrm{l}>>\mathrm{d}$, these shear components lead to the growth in the transversal directions but almost negligible change in the longitudinal directions. The complete process thus leads to a microscopically observed anisotropic growth effect. During the irradiation there are four different effects in which each manifests itself by characteristic changes in the mechanical stress state of the materials i.e., densification, stress relaxation by radiation-enhanced plastic flow, anisotropic expansion and stress generation and transient stress relaxation. These phenomena can cause the stress-induced cracks on the surface due to swift heavy ion irradiation. Moreover, the maximum momentum transfer, due to same atomic size of the incident ions and host material, leads to knockout (sputtering) of the silicon atoms towards the surface that may result in more stress on the surface. Stress induced cracks with a depth of $\sim 50 \mathrm{~nm}$ and width of $\sim 100 \mathrm{~nm}$ were found, however, the mechanism of the formation of cracks is not yet clearly understood. 
Nepal Journal of Science and Technology Vol. 14, No. 2 (2013) 155-160
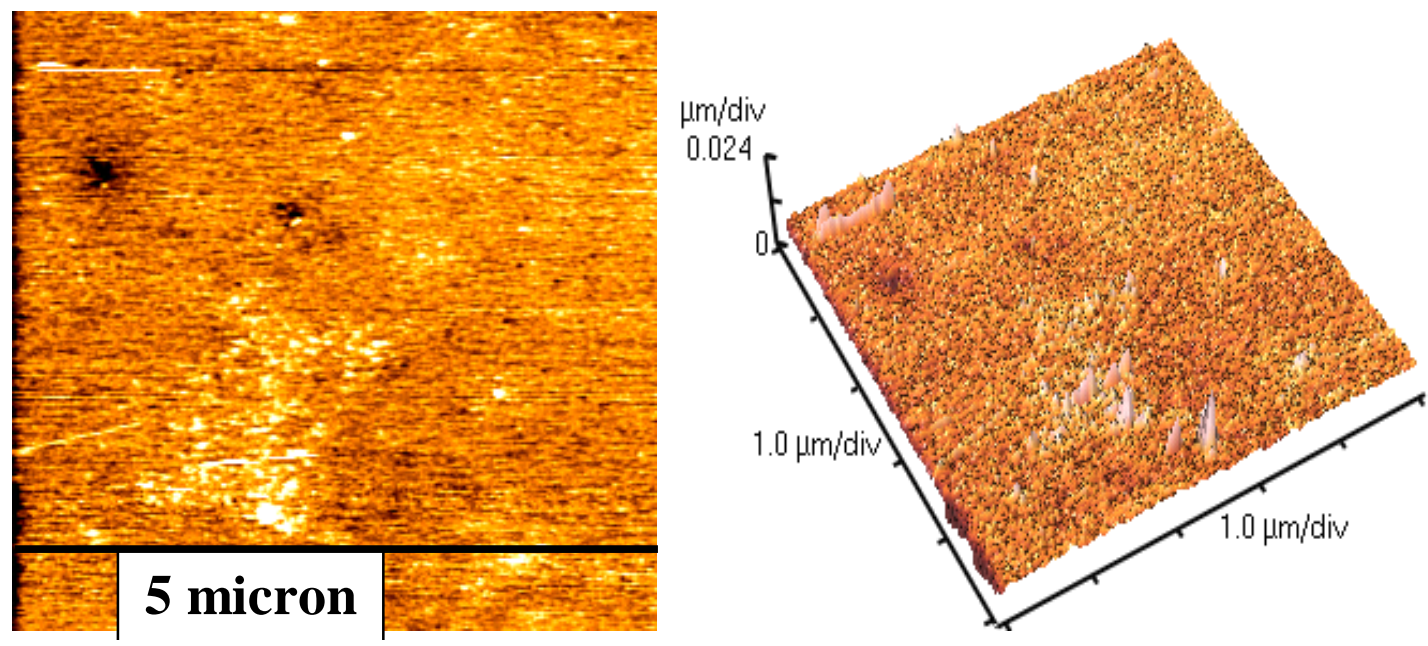

Fig. 1. AFM micrograph of an un-irradiated p-silicon surface, seems to be smooth and featureless.
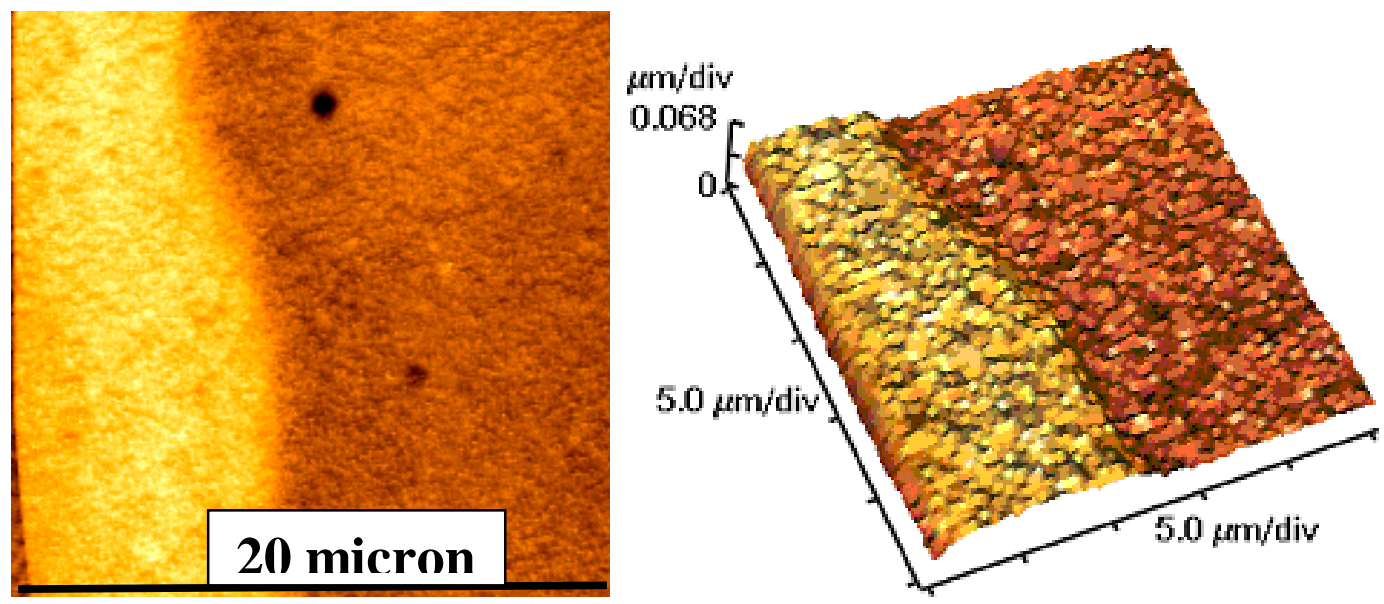

Fig. 2. AFM micrograph of p- Silicon surface irradiated with $\mathrm{MeV}$ ions to a fluence of $\sim 10^{11}$ ions $\mathrm{cm}^{-2}$.
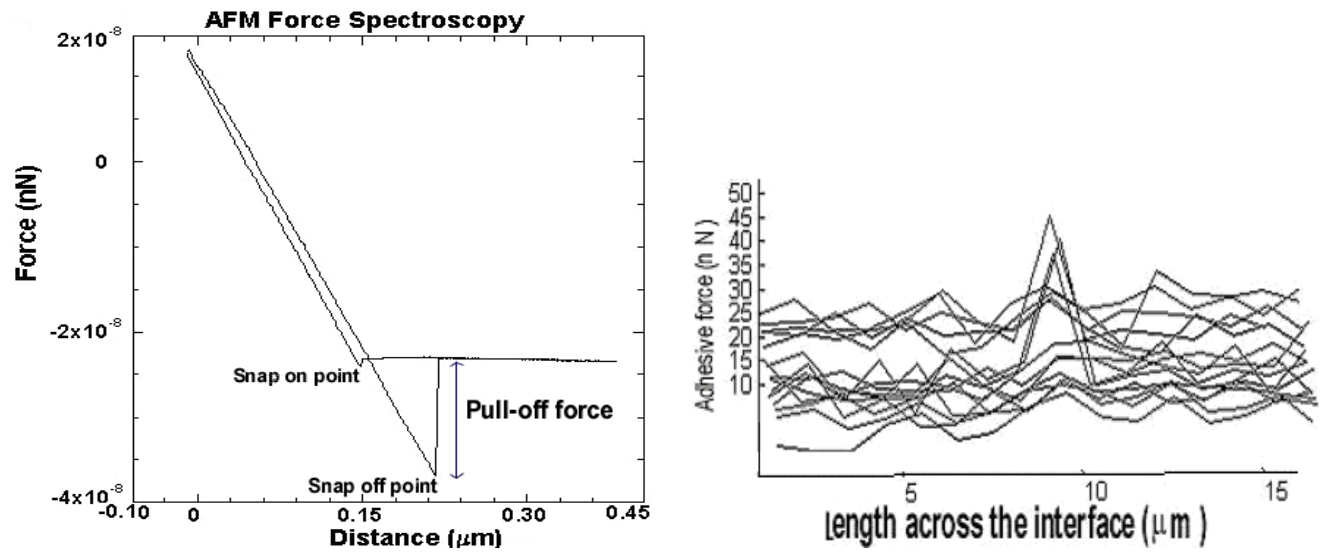

Fig. 3. A typical loading curve is shown. The adhesion of the tip is extracted by the loading curve. 
AFM has made its mark on wide varieties of applications, not only as a topographic mapping tool but also as a tool for studying surface interactions by means of force-distance curves, which has emerged since 1989. Force curves (force versus distance curve) typically relate to the deflection of the free end of the AFM cantilever, as its fixed end is brought vertically towards (approach) and then away from the sample (retraction) surface. This causes the scanner to expand and contract in the vertical direction generating relative motion between the cantilever and the sample as per atomic interaction between them. In this way, AFM generates and records the force felt by the cantilever, as the probe tip is brought close to and even indent into the sample surface and then pulled away. The force required to separate the two surfaces (the tip and the sample) is adhesive or pull-off force.

Force curve (Buldum et al. 1999) can be used to measure the pull-off or adhesion force. The adhesion force (Weisenhorn et al. 1993) depends on the nature of the binding interaction between the tip and surface during the contact. Adhesion maximum (Burnham \& Colton 1993) occurs where the tip has a larger contact area with the surface, while the adhesion minima are formed where the tip has the smaller contact area with surface. While moving a line across the interface from un-irradiated to irradiated part of the sample, we found that at the surface there was sharp increase in the adhesion force or there was adhesion maxima at the interface. We believe that this increase in adhesion force at the interface is due to compressive forces. These compressive forces have increased the force constant. The increased force constant brought atoms closer at the interface i. e. intra-atomic spacing has decreased at interface due to increase in contact area between the tip and surface. So, adhesion force increases at the interface.

In summary, properties response to a gradient is sensitive to the geometry of the microstructure and is the choice of path for energy transport. Generally, the behavior of phase depends on the characteristics of the adjacent grains and phase. The weighing procedures necessarily vary with the shape and distribution of the planes. Here we are having two phase microstructures. Results of AFM force spectroscopic study of radiation defects in silicon layers, $\mathrm{Si} / \mathrm{SiO}_{2}$ interface trap, along with oxide formation under irradiation with $\mathrm{MeV}$ in silicon-on- insulator (SOI) structures are presented and discussed. SOI was fabricated by the wafer bonding. It was found that electron irradiation led to transformation of energyspectrum of the interface traps (relaxation of the bonded interface) in SOI. The main effect of high-energy Kr ion irradiation consisted in formation of radiation defects in the top silicon layer as well as in the substrate.

\section{Acknowledgements}

Thanks for School of Physical Sciences, JNU for AFM characterization. Thanks for NSC, ND, India for irradiation experiment. Thanks for University Grants Commission, Kathmandu for financial support to the author.

\section{References}

Buldum, A., S. Ciraci, C. Y. Fong and J. S. Nelson. 1999. Interpretation of long range interatomic force. Phy. Rev. B 59: 5120-5125.

Burnham, N.A. and R. J. Colton.1993. Scanning tunneling microscopy and spectroscopy: theory, techniques and applications (Ed. D.A. Boneel). VCH, NewYork.

Erlandsson, R., G. Hadziioannou, C. M. Mcclelland, S. Chiang. 1988. Atomic scale friction between the muscovite mica cleavage plane and a tungsten tip. $J$. Chem. Phys. 89:5190 - 5194.

Koleske, D. D., G. U. Lee, B. I. Gans, K. P. Dilella, K. J. Wahl, W. R. Burger, L. J. Whitman and R. J. Colton. 1995. Design and calibration of a scanning force microscope for friction, adhesion, and contact potential studies, Rev. Sci. Instrum. 66: 4566 - 4575.

Lugomer, S. , A. Maksimoviæ, B. Farkas, Z. Geretovszky, T. Szörényi, A.L. Tóth, Z. Zolnai and I. Bársony. 2012. Multipulse irradiation of silicon by femtosecond laser pulses: Variation of surface morphology. Applied Surface Science 258 (8):3589 - 3597.

Martin, Y. and H.K. Wickramasinghe. 1987. Magnetic imaging by "force microscopy" with $1000 \AA$ resolution, Appl Phys. Lett. 50:1455 - 1458.

Mizes, H.A., K. G. Loh, R. J. D. Miller, S. K. Ahuja and E. F. Grabowski.1991.

Sen, P. and J. Akhtar. 2002. Nonequilibrium processes for generating silicon nanostructures in single-crystalline silicon. Pure and Appl. Chem. 74 (9):1631-1641.

Sen, P., G. Aggarwal and U. Tiwari. 1998. Dissipative structure formation in cold-rolled Fe and Ni during heavy ion irradiation. Phys. Rev. Lett. 80: 97-100.

Sen, P., J. Akhtar and F. M. Russel. 2000. MeV ioninduced movement of lattice disorder in single crystalline silicon. Europhys. Letters 51 (4):401405. 
Nepal Journal of Science and Technology Vol. 14, No. 2 (2013) 155-160

Mizes, H.A., K. G. Loh, R. J. D. Miller, S. K. Ahuja and E. F. Grabowski.1991.Submicron probe of polymer adhesion with atomic force microscopy: Dependence on topography and material inhomogeneities. Appl. Phys. Lett. 59: 2901 - 2903.
Weisenhorn, A. L., M. Khorsandi, S. Kasas, V. Gotzos and H. J. Butt. 1993. Deformation and height anomaly of soft surfaces studied with an AFM. Nanotechnology 4: 106 - 110. 